NBER WORKING PAPER SERIES

\title{
A TRANSACTIONS BASED MODEL OF THE MONETARY TRANSMISSION MECHANISM: PART 2
}

\author{
Sanford J. Grossman
}

Working Paper No. $97 \underline{4}$

\author{
NATIONAL BUREAU OF ECONOMIC RESEARCH \\ 1050 Massachusetts Avernue \\ Cambridge MA 02138 \\ September 1982
}

The research reported here is part of the NBER's research programs in Economic Fluctuations and in Financial Markets and Monetary Economics. Any opinions expressed are those of the author and not those of the National Bureau of Economic Research. 
In Part 1 the dynamics of an open market operation were analyzed for the case of logarithmic utility. Though such a utility function is useful for illustrative purposes, the implication that current prices are independent of current and future monetary injections is unsatisfactory. This implication results from the fact that with logarithmic utility future consumption is independent of the rate of return to savings. In Part 2 the logarithmic utility assumption is replaced by the more general assumption that utility is of the constant elasticity form such that future consumption is an increasing function of the interest rate. Though a closed form solution cannot be derived for this case, it is shown that the basic results of Part 1 still hold: An increase in money causes a sluggish response of the price level and a fall in interest rates.

Professor Sanford Grossman University of Chicago Department of Economics 1126 E. 59th Street Chicago, Illinois 60637

(312) $962-8178$ 


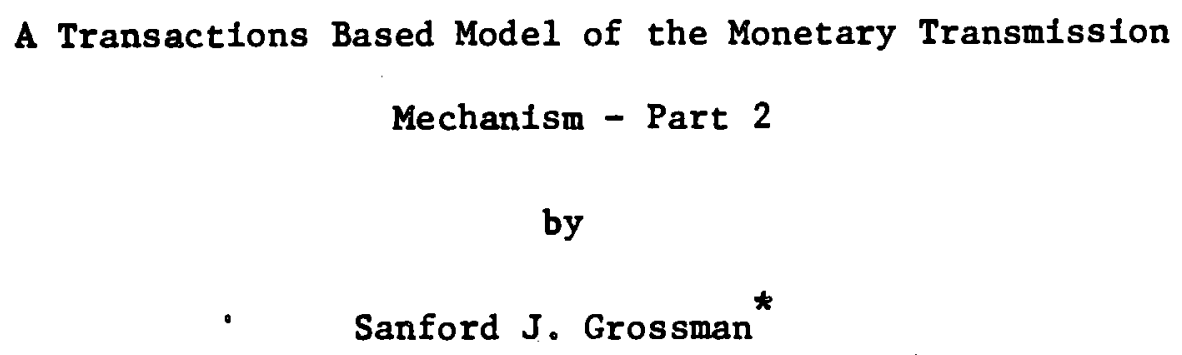

In Part 1 the dynamics of an open market operation were analyzed for the case of logarithmic utility. Though such a utility function is useful for illustrative purposes, the implication that current prices are independent of current and future monetary injections is unsatisfactory. This implication results from the fact that with logarithmic utility future consumption is independent of the rate of return to savings. In Part 2 the logarithmic utflity assumption is replaced by the more general assumption that utility is of the constant elasticity form such that future consumption is an increasing function of the interest rate. Though a closed form solution cannot be derived for this case, it is shown that the basic results of Part 1 still hold: An increase in money causes a sluggish response of the price level and a fall in interest rates.

\section{The Basic Difference Equation}

This Part refers freely to the equations in Part 1. Assume that

$$
u(c)=\frac{c^{1-A}}{1-A}
$$

Then from $(2.12 a)$ savings $\phi(X, Y)$ can be written as

\footnotetext{
Untverstiy of Chicago I am grateful to Jose Scheinkman for helpful advice. All errors are mine. Research supported by NSF Grant SES-8112036.
} 


$$
\phi(X, Y)=Y \phi(X) \text {, where } \phi(X)=\frac{\beta^{1 / A}}{\beta^{1 / A}+\frac{A-1}{X}}
$$

Assume that $0<A<1$, so that $\phi^{\prime}(X)>0$, 1.e., future consumption is an increasing function of the interest rate. The case $A=1$ was the concern of Part 1. Note that $0<\phi(X)<1$ for $0<\mathrm{X}<\infty$.

As in Part 1 we will be concerned with equillbrium near the steady state, where the initial cash in advance constraint will be binding. In order to analyze an open market operation which occurs only during period 1 , set

$$
M_{t}^{s}=M(1+k) \text {, for } t \geq 1 \text {, }
$$

where $k$ is the \% increase in money which occurs at time 1 , and $M=M_{0}^{a}+M_{0}^{b}$ is the steady state stock of money. Also set $y_{t} \equiv y_{0}$ Thus (2.20), gives the following second order difference equation

$$
p_{t} y+\phi\left(\frac{p_{t}}{p_{t+1}}\right) \quad p_{t-1} y=(1+k) M \text { for } t \geq 3,
$$

and (2.22) gives the constraints on initial conditions

$$
\begin{aligned}
& \mathrm{p}_{1} \mathrm{y}+\phi\left(\frac{\mathrm{p}_{1}}{\mathrm{p}_{2}}\right) \mathrm{M}_{0}^{\mathrm{b}}=\mathrm{M} \\
& \mathrm{p}_{2} \mathrm{y}+\phi\left(\frac{\mathrm{p}_{2}}{\mathrm{p}_{3}}\right)\left(\mathrm{p}_{1} \mathrm{y}+\mathrm{kM}\right)=(1+\mathrm{k}) \mathrm{M} .
\end{aligned}
$$


Recall that for $t \geq 3, p_{t-1} y$ is the amount of money flowing into and hence out of the bank at $t-1$. Thus $\phi\left(\frac{p_{t}}{p_{t+1}}\right) p_{t-1} y$ is the money holdings at the end of $t$ for someone who went to the bank at the end of $t-1$, and thus plans to exhaust his money at the end of $t+1$ by spending $\phi\left(\frac{p_{t}}{p_{t+1}}\right) p_{t-1} y$ during $t+l$. The term $\Gamma_{t} y$ is the money held at the end of $t$ by the people making a withdrawal at $t$. Hence the left hand side of (2) is total money holdings at the end of $t$.

Note that (2) implies that

$$
\dot{p}_{t}+\phi\left(\frac{p_{t}}{p_{t+1}}\right) p_{t-1}=p_{t+1}+\phi\left(\frac{p_{t+1}}{p_{t+2}}\right) p_{t} .
$$

Let $\gamma_{t} \equiv \frac{P_{t}}{P_{t+1}}$, then (4) can be written as

$$
\phi\left(\gamma_{t+1}\right)=\phi\left(\gamma_{t}\right) \gamma_{t-1}+1-\frac{1}{\gamma_{t}} \text { for } t \geq 3 .
$$

Since prices are non-negative, (5) is defined only for non-negative $\gamma_{t}$.

Equation (5) gives the path of the one period returns to holding money $Y_{t}$. It is somewhat easier to work with rates of return than prices so we will study (5) rather than (2). Note that (5) has only one steady state namely $\gamma_{t}=\gamma_{t-1}=1$. This can be seen by writing (5) as

$$
\phi(\gamma)-\phi(\gamma) \gamma=1-\gamma^{-1} \text {, or, } \phi(\gamma)(1-\gamma)=1-\gamma^{-1} \text {. }
$$

This last equation can only hold for $\gamma=1$, since $0<\phi<1$. We will use the following Lemma: 
Lemma 1. (a) If $\gamma_{2}<1$ and $\gamma_{3}<1$, then there exists an $\varepsilon>0$ such that $\gamma_{t}<1-\varepsilon$ for all $t \geq 2$. (b) If $\gamma_{2}>1$ and $\gamma_{3}>1$ then there exists an $\varepsilon>0$ such that $\gamma_{t}>1+\varepsilon$ for all $t \geq 2$. In both cases (5) will eventually fail to hold, i.e., $0 \leq \phi(\gamma) \leq 1$ will be violated.

Proof. (a) From (5), $\phi\left(\gamma_{4}\right)<\phi\left(\gamma_{3}\right) \gamma_{2}<\phi\left(\gamma_{3}\right)$. Hence, since $\phi^{\prime}(\gamma)>0$ $\gamma_{4}<\gamma_{3}<1$. Similarly $\phi\left(\gamma_{5}\right)<\phi\left(\gamma_{4}\right) \gamma_{3}<\phi\left(\gamma_{4}\right)$ so $\gamma_{5}<\gamma_{4}<\gamma_{3}<1$. It is easy to see by induction that (i) $\gamma_{t-1}<1$ and $\gamma_{t}<1$ implies that $\gamma_{t+1}<1$ and $\gamma_{t+2} \leqslant 1$, and (ii) $\gamma_{t+1}<\gamma_{t}$. Hence since $\gamma_{3}<1$, the $\gamma_{t}$ are bounded away from 1 .

(b) The signs of all the above reverse, i.e., $\gamma_{t-1}>1 \gamma_{t}>1$ implies from (5) that $\phi\left(\gamma_{t+1}\right)>\phi\left(\gamma_{t}\right) \gamma_{t-1}>\phi\left(\gamma_{t}\right)$ so $\gamma_{t+1}>\gamma_{t}$, etc.

In both cases a monotone $\gamma_{t}$ sequence is generated which diverges from the unique steady state $\gamma=1$. Hence $\gamma_{t}$ must go to positive infinity or zero. However this will violate (5) for $\gamma_{t}$ sufficiently large or close to zero. QED

Lemma 1 implies that if there are ever 2 consecutive $\gamma_{t}$, both above 1 or both below 1 then $p_{t}$ goes to infinity or $p_{t}$ goes to zero, and (5) will eventually fail. Each of these possibilities is inconsistent with market clearing. To see this look at equation (2). Since $k$ and $M$ are given with $(1+k) M>0$ and $0 \leq \phi \leq 1$, it cannot be the case that prices get indefinitely high or go to zero: (2) will eventually fall. Intuitively, if prices get too high then people will be demanding more money than the whole stock of money to make their purchases. Similarly if $p_{t}$ gets too small the purchasing power of $M(1+k)$ will exceed the stock of goods. Hence 
Corollary 1. If $P_{t}$ is an equilibrium price path $1 . e .$, (2) and (3) hold, then there is no $t \geq 3$ such that (a) $\gamma_{t}<1$ and $\gamma_{t+1}<1$, or (b) $\gamma_{t}>1$ and $\gamma_{t+1}>1$

Recall from Part 1, that except for the case of logarithmic utility we have no equation to determine $p_{1}$. That is, (3a) gives $p_{2}$ as a function of $p_{1}$. This and (3b) gives $p_{3}$ as a function of $p_{1}$. Thus $p_{3}$ and $p_{2}$ are determined as a function of $p_{1}$. Equation (2) is a second order difference equation which requires $a p_{3}$ and $p_{2}$ to start it off. Thus for every $p_{1}$, in general there will be a path generated by (2) and (3) which is a candidate path for an equilibrium. The paradox of this seemingly continuum of equilibria can be resolved by showing that the second order difference equation in (2) will eventually violate $0 \leq \phi \leq 1$ unless $p_{2}$ and $p_{3}$ satisfy a particular functional relationship. In particular given a $p_{2}$ we will show that there exists at most one $p_{3}$ such that (2) can hold for all $t \geq 2$ and $0 \leq \phi \leq 1$.

Recall that (5) has a unique steady state $\gamma=1$. We will assume that for each $\gamma_{2}$ there exists some $\gamma_{3}$ such that the $\gamma_{t}$ generated by (5) converge to 1 . We will show that this implies that there is at most one $\gamma_{3}$ for each $\gamma_{2}$ such that (5) holds for each $t \geq 3$. After proving the above statement we will show that the hypothesis is not empty. That is, there exists a neighborhood of 1 such that for any $\gamma_{2}$ in that neighborhood there exists a $\gamma_{3}$ such that the solution to (5) converges to the steady state. We will need the following theorem. 
Theorem 1. Let $g(x)$ be a continously differentiable function from $\mathbf{R}^{2}$ to $\mathbb{R}^{2}$. Consider the difference equation $x_{t+1}=g\left(x_{t}\right)$ with a steady state $\bar{x}$, 1.e., $x=g(\vec{x})$. Consider the Iinearized difference equation about $\bar{x}$ : $x_{t+1}-\bar{x}=\bar{A}\left(x_{t}-\bar{x}\right)$, where $\bar{A}=\nabla g(\bar{x})$ is the $2 \times 2$ matrix of derivatives of 8 with respect to $x$. Assume that the two characteristic roots of A satisfy $\left|\lambda_{1}\right|>1$ and $\left|\lambda_{2}\right|<1$. Then there exists a one dimensional manifold $M$ in $\mathbb{R}^{2}$, tangent to the stable manifold of the linear system at $\bar{x}$, with the following properties: there exists an open neighborhood of $\bar{x}$ say $N$ such that if $x_{0} \in N \cap M$ and $\left\{x_{t}\right\}$ is generated from $x_{t+1}=g\left(x_{t}\right)$ starting at $x_{0}$, then $x_{t} \rightarrow \bar{x}_{0}$ Further if $x_{0} \varepsilon N$ and $x_{0} \notin M$ then there exists a time $t$ such that $x_{t} \$ N$.

Theorem 1 is analogous to the stable manifold theorems for differential equations, see Coddington and Levinson Theorems 4.1 and 4.2 on pp.330-334. of course, not all theorems for differential equations are true for difference equations. However Theorem 1 is true. In particular Scheinkman (1974) (i.e., his Lemma 6 of Part II) proves most of Theorem 1 following steps analogous to Coddington and Levinson. The remainder of the theorem is straightforward following Scheinkman's discrete time rendition of Coddington and and Levinson. Thus a proof will not be given here.

We will use Theorem 1 to prove a global property of the difference equation (5).

Theorem 2. Let $\gamma_{2}=\bar{\gamma}_{2}$ be given. Assume that there exists a $\bar{\gamma}_{3}$ such that the solution to (5) converges. Then for $\gamma_{2}=\bar{\gamma}_{2}, \bar{\gamma}_{3}$ is the unique value for $\gamma_{3}$ for which (5) can hold for all $t>2$ without violating $0 \leq \phi(\gamma) \leq 1$, i.e., for which there exists a solution path to (5). 


\section{Proof.}

We must show that there is no path $\gamma_{t}$ satisfying (5) starting from $\gamma_{2}=\bar{\gamma}_{2}$ and $\gamma_{3} \neq \bar{\gamma}_{3}$. We will denote the convergent path starting from $\left(\bar{\gamma}_{2}, \bar{\gamma}_{3}\right)$ by $\bar{\gamma}_{t}$. We first show that it is impossible for $\gamma_{3}>\bar{\gamma}_{3}$. If $\gamma_{3}>\bar{\gamma}_{3}$, then from (5)

$$
\phi\left(\gamma_{4}\right)=\phi\left(\gamma_{3}\right) \gamma_{2}+1-\frac{1}{\gamma_{3}}>\phi\left(\bar{\gamma}_{3}\right) \gamma_{2}+1-\frac{1}{\bar{\gamma}_{3}}=\phi\left(\bar{\gamma}_{4}\right),
$$

so $\gamma_{4}>\bar{\gamma}_{4}$. It is easy to see by induction that $\gamma_{t}>\bar{\gamma}_{t}$ for all $t$. Further, by Lemma 1 , if $\gamma_{t}$ satisfies (5) then $\gamma_{t}-1$ must alternate in sign as $t$ goes to. $t+1$. Note that either $\gamma_{t}$ converges to 1 or it does not.

First suppose that $\gamma_{t}$ converges to 1 . Then by Theorem 1 , there exists a neighborhood of $(1,1)$ so that $\left(\gamma_{t}, \gamma_{t-1}\right)$ will be on the stable manifold of (5) "near" where it is tangent to the stable manifold of the linearized system. More precisely write (5) as

$$
x_{t+1}=g\left(x_{t}\right), \quad t \geq 3
$$

where

$$
x_{t} \equiv\left(\gamma_{t}, \gamma_{t-1}\right) \quad g\left(x_{t}\right) \equiv\left(\phi^{-1}\left(\phi\left(\gamma_{t}\right) \gamma_{t-1}+1-\gamma_{t}^{-1}\right), \gamma_{t}\right) .
$$

Note that $g(x)=\bar{x}$ has a single solution in $R_{+}^{2}, \bar{x}=(1,1)$. The eigenvalues of $\bar{A}=\nabla g(\bar{x})$ are given by

$$
\lambda_{1}=\frac{2 \phi}{-\left(\phi^{\prime}+1\right)+\sqrt{\left(\phi^{\prime}+1\right)^{2}+4 \phi \phi^{\prime}}}
$$

$$
\lambda_{2}=\frac{2 \phi}{-\left(\phi^{\prime}+1\right)-\sqrt{\left(\phi^{\prime}+1\right)^{2}+4 \phi \phi^{\prime}}}
$$

where $\phi \equiv \phi(1)$ and $\phi^{\prime} \equiv \phi^{\prime}(1) .1^{\prime} \cdot \operatorname{Recal1}$ that $\phi^{\prime}>0$ and $0<\phi<1$. It can be shown that $-1<\lambda_{2}<0$ and $\lambda_{1}>1$. Therefore the stable mantfold 
of the linear system (starting at $t \geq 3$ ) satisfies

$$
\left(\gamma_{3}-1, \gamma_{2}-1\right) \cdot\left(1,-\lambda_{2}\right)=0 \text { or } \gamma_{3}-1=\lambda_{2}\left(\gamma_{2}-1\right)
$$

Thus the stable linear manifold has a negative slope in $\left(\gamma_{t}, \gamma_{t-1}\right)$ space. Hence by Theorem 1 , the stable mantfold of the non-linear system (6) has a negative slope in some neighborhood of $(1,1)$. Denote the stable manifold of the nonlinear system by $\gamma_{t}=m\left(\gamma_{t-1}\right)$. Since $\gamma_{t}$ and $\bar{\gamma}_{t}$ both converge to 1 , for $t$ large enough $\gamma_{t}=m\left(\gamma_{t-1}\right)$ and $\bar{\gamma}_{t}=m\left(\bar{\gamma}_{t-1}\right)$. Recall that for all $t \quad \gamma_{t}>\bar{\gamma}_{t}$. Hence $\gamma_{t-1}>\bar{\gamma}_{t-1}$, but for $t$ large enough $m(\gamma)$ has a negative slope, so $m\left(\gamma_{t}\right)<m\left(\bar{\gamma}_{t}\right)$. Hence $m\left(\gamma_{t}\right)=\gamma_{t+1}<\bar{\gamma}_{t+1}=m\left(\bar{\gamma}_{t}\right)$ which is impossible. This shows that $\gamma_{t}$ does not converge to 1 .

Suppose that $\bar{\gamma}_{t}<1$. Then by Lemma $1 \bar{\gamma}_{t+1}>1$. Further it must be the case that $\gamma_{t}<1$, for if $\gamma_{t}>1$ then $\gamma_{t+1}<1$ which contradicts $\gamma_{t+1}>\bar{\gamma}_{t+1}$. Thus for every other value of $t, \bar{\gamma}_{t}<\gamma_{t}<1$, with $\bar{\gamma}_{t} \rightarrow 1$. Without loss of generality we may assume that along, say, even values of $t$ $\gamma_{t}$ converges to 1 from below. Further, since $\gamma_{t}$ does not converge to 1 , for some $\varepsilon>0$ and any $\delta>0$ there exists an even $t$ such that $1-\gamma_{t}<\delta$ and $\gamma_{t-1}>1+\varepsilon$. But this will violate (5), since for $\delta$ sufficiently small (5) will imply $\phi\left(\gamma_{t+1}\right)>1$. This shows that it is impossible for $\bar{\gamma}_{3}<\gamma_{3}$. A similar argument shows that $\bar{\gamma}_{2}>\gamma_{2}$ is impossible. QED

Let $S$ be the set of $\gamma_{2}$ such that there exists a $\gamma_{3}$ with the property that when (5) is started at $\left(\gamma_{2}, \gamma_{3}\right)$ then a solution for $\gamma_{t}$ exists for all $t \geq 2$. In the proof of Theorem 2 it was shown that the set $S$ is not empty. This is because it was shown that the linearized system has a non-degenerate stable manifold. Thus $S$ contains an open neighborhood of 1 . Theorem 2 implies that if $\gamma_{2} \varepsilon s$ then there exists 
a unique $\gamma_{3}$ for which (5) holds. We denote this $\gamma_{3}$ by $\mathrm{m}\left(\gamma_{2}\right)$, i.e., $\gamma_{3}=m\left(\gamma_{2}\right)$.

Returning to the equations involving $p_{t}$ recall from Part 1 that we did not describe how $p_{1}$ is chosen except for the logarithmic case. We can use the function $m(\cdot)$ to determine $p_{1}$ as follows. Given $p_{1}$ determines $\gamma_{1}$ as a function of $p_{1}$ and $k$, say $\gamma_{1}\left(p_{1}, k\right)$. Hence $p_{2}=p_{1} \div \gamma_{1}\left(p_{1}, k\right)$. Thus (3b) determines $\gamma_{2}$ as a function of $p_{1}$ and $k$, say $\gamma_{2}\left(p_{1}, k\right)$. Next apply equation (2) at $t=3$ to get $\gamma_{3}\left(p_{1}, k\right)$. Then $p_{1}$ must be chosen so that

$$
\gamma_{3}\left(p_{1}, k\right)=m\left(\gamma_{2}\left(p_{1}, k\right)\right)
$$

holds. By differentiating the above functions and evaluating at $k=0$, it is easy to see that there is a unique $p_{1}$ such that (8) holds for $p_{1}$ in a neighborhood of the steady state $p=M_{0}^{b} \div y \cdot \underline{2}$ I

II. The Non Neutrality of Open Market Operations

It is easy to use Lemma 1 to show that prices respond slowly to an open market operation. The next Theorem shows that a $k \%$ increase in money via an open market operation leads the initial price to move by less than k\%. (Similarly prices initially fall by less than $k \%$ if there is a monetary contraction.) Throughout this Section $\mathrm{M}_{0}^{a}$ and $\mathrm{M}_{0}^{\mathrm{b}}$ are as given in (3.2).

Theorem 3. If $M \equiv M_{0}^{a}+M_{0}^{b}>0$, and $M_{t}^{S}=(1+k) M>0$ for $t \geq 1$, then $p_{1}<(1+k) p$ when $k>0$, and $p_{1}>(1+k) p$ when $k<0$, where $p$ is the steady state price level when $k=0$. 
Proof.

We give the proof for $k<0$. The proof for $k>0$ is similar. So suppose $\mathrm{k}<0$ and $\mathrm{p}_{1}<(1+\mathrm{k}) \mathrm{p}$, then this leads to a contradiction as follows. Let $p_{t}$ be the equilibrium path generated for $k<0$. From (3a)

$$
\phi\left(\gamma_{1}\right)=\frac{M-p_{1} y}{M_{0}^{b}} \geq \frac{M-(1+k) p y}{M_{0}^{b}}>\frac{M-p y}{M_{0}^{b}}=\phi(1),
$$

where the last equality follows from (3.2). Hence $\gamma_{1}>1$. Hence $\mathrm{p}_{2}<(1+\mathrm{k}) \mathrm{p}$. Recall that the steady state for $\mathrm{k}=0$ satisfies py $+\phi(1) p y=M . \quad$ Thus

$$
(1+k) p y+\phi(1)(1+k) p y=(1+k) M .
$$

Hence from (3b) $\phi\left(\gamma_{2}\right)>\phi(1)$, so $\gamma_{2}>1$. Hence $\mathrm{p}_{3} \mathrm{y}<(1+\mathrm{k}) \mathrm{py}$. Apply this to (2) evaluated at $t=3$ and conclude that $\phi\left(\gamma_{3}\right)>\phi(1)$, so $\gamma_{3}>1$. By Corollary 1 this is impossible.

QED

It can also be shown that prices respond gradually to a small monetary injection, with $\mathrm{p}_{1}$ rising, $\mathrm{p}_{1} / \mathrm{p}_{2}, \mathrm{p}_{2} / \mathrm{p}_{3}$ falling and $\mathrm{p}_{3} / \mathrm{p}_{4}$ rising relative to their steady state positions of $p, 1,1$, and 1 respectively. This is easily seen by differentiating (8) with respect to $k$, evaluating the derivatives at $k=0$, and using the fact that $\mathrm{m}^{\prime}(1)=\lambda_{2}$ is the slope of the linearized system in Theorem 2 .

Finally, it is possible to show that a small increase in money by an open market operation lowers both the initial two period nominal rate and real rate. This can be proved by noting that from (2.23) 


$$
\begin{array}{r}
u^{2} R_{1} R_{2}=\frac{1}{\gamma_{2} \gamma_{3}} u^{\prime}\left(y-\frac{\phi\left(\gamma_{1}\right) M_{0}^{b} \gamma_{1}}{\left(y-\phi\left(\gamma_{3}\right) \gamma_{2} \gamma_{3} y\right)}\right. \\
\left.P_{1}\right)
\end{array}
$$

Recall that equation ( 8 ) determines $p_{1}$ as a function of $k$, and hence determines $\gamma_{2}$ and $\gamma_{3}$ as a function of $k$. Thus $R_{1} R_{2}$ is determined as a function of $k$. This function can be differentiated and evaluated at $k=0$ to verify that an increase in $k$ lowers $R_{1} R_{2}$ for $k$ small. Similarly the real race is easily shown to fall because there is inflation from $t=2$ to $t=4$, i.e. $p_{4} \div p_{2}=\left(\gamma_{2} \gamma_{3}\right)^{-1}$ has a positive derivative with respect to $k$ at $k=0$.

\section{Conclusions}

The determination of the initial price level for the model of Part 1 with non-logarithmic utility has been presented. This facilitated a simple proof that open market increases in money lead to a sluggish price level response and a temporary fall in interest rates. These results assume that the open market operation is sufficiently small so that people do not return to the bank (initially) with unspent cash. Throughout Part 1 and Part 2, the period between trips to the bank has been taken to be independent of the size of the open market operation. It would be useful but difficult, to extend our results to a model where the transaction period is endogenous. It would be far more useful to discuss the possibility of returns to the bank with unspent cash balances in such a model, rather than in the current model. 


\section{Footnotes}

$\underline{1}$
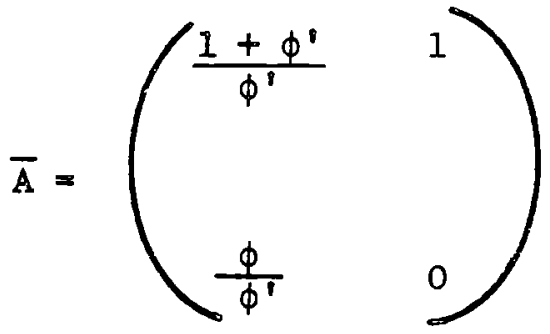

The eigenvector corresponding to

$$
\lambda_{1} \text { is }\left(\begin{array}{c}
1 \\
-\lambda_{2}
\end{array}\right) \text {. }
$$

2/ This shows that there is a unique equilibrium for economies beginning near $k=0$ (i.e., for small monetary shocks), given that the initial cash in advance constraints are binding. Clearly, if we do not assume they are binding there will be no $p_{1}$ in a neighborhood of the $p_{1}$ which solves ( 8 ) in which the constraint will fail to bind, and which leads to a convergent equilibrium. This is because, by the stable manifold property small changes in the initial conditions will lead to small changes in all $\gamma_{\text {f }}$ and consumptions. Thus since (2.26) holds as a strict inequality for the solution to (8) it will also be a strict inequality for the alternative path. Hence the cash in advance constraint will bind for all prices which begin in a neighborhood of the steady state.

\section{References}

1. Coddington, E. and N. Levinson, Theory of Ordinary Differential Equations, McGraw-Hill, New York, 1955.

2. Scheinkman, J., "On Optimal Steady States of n-Sector Growth Models When Utility is Discounted", Unpublished Doctoral Dissertation, Department of Economics, University of Rochester, 1974.

3. Grossman, S. and L. Weiss, "A Transaction Based Model of the Monetary Transmission Mechanism - Part I". 